\title{
Studies on the physicochemical properties of Abelmuscus esculentus L. (Okra) pods - a potential tablet excipient
}

\author{
Lateef GBENGA BAKRE * and Kolawole T. JAIYEOBA
}

Department of Pharmaceutics and Industrial Pharmacy, Faculty of Pharmacy, University of Ibadan, Nigeria. *Corresponding author, E-mail: lateefbakr@yahoo.com,Tel.: 234-8033700102.

\begin{abstract}
This study aims at evaluating the physicochemical and compaction characteristics of dried powdered pods of Abelmuscus esculentus (Okra). The physicochemical properties were determined and the compressional behaviour of the Okra powder was analysed using density measurements and parameters from Heckel and Kawakita plots. Okra powders had higher tapped and bulk density values than microcrystalline cellulose but lower values compared to corn starch. The hydration and swelling capacities values showed that Okra powders were capable of absorbing at least five times its own weight of water and swelled considerably. Furthermore, Okra powders had superior hydration and swelling capacities $(\mathrm{p}<0.05)$ to corn starch and microcrystalline cellulose. The ability to absorb water and swell considerably could be explored in disintegration of tablets. Corn starch induced faster onset of plastic deformation than Okra powders but the overall amount of plastic deformation occurring during compression was higher for Okra powders. Therefore tableting machines with longer dwell times will be more appropriate for the production of tablets formulated with Okra powders. Results suggest that Okra powder has great potentials as pharmaceutical raw material in tablet formulation and production.

(C) 2009 International Formulae Group. All rights reserved.
\end{abstract}

Keywords: Okra, hydration capacity, swelling capacity, compressional characteristics.

\section{INTRODUCTION}

Pharmaceutical oral solid dosage forms have been used widely for decades mainly due to the convenience of administration and suitability for delivery of drugs for systemic effects. The most commonly used pharmaceutical solid dosage forms today include tablets and capsules. The ease of manufacturing, convenience of administration, accurate dosing, and relative stability compared to oral liquids, tamper - proofness compared to capsules, safety compared to parenteral dosage forms makes tablet a popular and versatile dosage form (Gohel and Jogani, 2005). Tablets can be made directly from powders or via intermediates such as granules and pellets. Powdered drugs are generally not used alone when formulating solid dosage forms. A variety of excipients such as diluents, disintegrants, binding agents and lubricants; the vast majority of which are in powder form, are included for particular functions. They are then processed in convenient forms for drug administration. While full chemical profiles of drugs and excipients are generally well defined for quality assurance purposes, it is important to characterize their fundamental powder and processing properties, since in principle all factors influencing the final properties of the compact depend upon them (Leuenberger and Rohera, 1986). In addition, information on the compressibility, one of the physicotechnical properties used in characterizing powder materials is extremely important and it is defined as the ability of the material to decrease in volume under pressure (Patel et al., 2006). 
Okra is an annual or perennial herbaceous plant, growing up to $2 \mathrm{~m}$ tall, straight up with very little phototropism. The pod could be green, red or purple, long, slender or chunky with numerous ridges running along the length of the pod. The optimum conditions for storing Okra are a moist environment and temperatures of 7 to $10{ }^{\circ} \mathrm{C}$ (Smith et al., 2006). Okra is normally eaten young (in soups and as a vegetable) as it gets very woody when mature.

This study aims to evaluate the physicochemical and compaction properties of dried powdered pods of Abelmuscus esculentus (Okra) with a view to providing information on its potential usefulness as pharmaceutical raw material in tablet formulation and production.

\section{MATERIALS AND METHOD Materials}

The materials used were: Corn starch $\mathrm{BP}$ and microcrystalline cellulose (BDH Chemical, Poole, UK), benzene (Hopkin and Williams, London) and magnesium stearate (Caelo GmbH, Germany). Okra powder was prepared in the Pharmaceutics laboratory, University of Ibadan, Nigeria and water was double distilled.

\section{Methods}

\section{Preparation of Okra pod powders}

A $5.0 \mathrm{~kg}$ weight of fresh Okra pods from which the stalk and the apex of the pod had been removed was weighed, sliced with a hand knife and spread in the sun for 72 hours. The sun drying was done between 9:00 hrs and 16:00 hrs daily. The average temperature during this period was $33{ }^{\circ} \mathrm{C}$ and the relative humidity was $67 \%$. The sun dried pods were then crushed into tiny bits in a mortar and then pulverized in an osterizer blender to produce powdered Okra labeled as FOP-SD. Fresh Okra pods without the apex and the stalk, over ripped pods (pods that have become tough and fibrous due to delay in harvesting) without the apex and the stalk, the stalk and the apex were prepared as above but dried in a Gallenkamp oven (Model BS, UK) at $60{ }^{\circ} \mathrm{C}$ for 9 hours to produce powdered Okra labeled as FOP-OV, TOP-OV and OST-OV respectively

\section{Evaluation of physicochemical properties of Okra powders}

The particle (true) density was determi- ned by the pycnometer method using the liquid immersion technique with benzene as the displacement fluid. The bulk density of each powder at zero pressure (loose density) was determined by pouring $25 \mathrm{~g}$ of the powder at an angle of $45^{\circ}$ through a funnel into a glass measuring cylinder with a diameter of $21 \mathrm{~mm}$ and a volume of $50 \mathrm{ml}$. The bulk density was calculated from the ratio of the weight of the sample to the bulk volume. This was carried out in triplicate and the final bulk density was the mean determination of the three values. Tap density was determined by subjecting the powder in a graduated cylinder to 300 taps using a standardized procedure of 38 taps per minutes (British Standard 1460, 1970). The precompression density $\left(D_{o}\right)$ was calculated from the ratio of the loose bulk density to the particle density.

The hydration capacity was determined by placing $400 \mathrm{mg}$ samples in each of $15 \mathrm{~mL}$ plastic centrifuge tubes, distilled water was added, the tubes were covered with parafilm and the contents mixed on a vortex mixer for 2 minutes. The mixture in each tube was left for an additional 3 minutes and then centrifuged at $3000 \mathrm{~g}$ on a centrifuge (Griffin and George, U.K). The supernatant was decanted and the sediment weighed. The weight of water absorbed and retained was determined as the gain in weight of dry sample from equation (1). Determinations were made in triplicate.

$H C=\frac{(\text { weightof tube }+ \text { sediment })-\text { weight of tube }}{\text { Sample weight }(\text { dry basic })}$

HC: Hydration Capacity

The moisture sorption capacity was determined by weighing 2 g quantity of Okra powder. This was distributed evenly over the surface of a $70 \mathrm{~mm}$ tarred Petri dish and placed in a large desiccator containing distilled water in its reservoir $(\mathrm{RH}=100 \%)$. The desiccator was stored at room temperature at various time intervals over a five - day period. The weight gained by the exposed sample was recorded and the amount of water absorbed was calculated from the weight difference.

The swelling capacity was obtained by putting $3 \mathrm{~g}$ of the Okra powder in a $50 \mathrm{ml}$ ground-glass stoppered graduated cylinder 
and the volume occupied, $\mathrm{V}_{\mathrm{O}}$, was noted. 20 $\mathrm{ml}$ of water was added and the cylinder closed. This was shaken vigorously every 10 minutes for 1 hour and then allowed to stand for 5 hours. At 2.5 hours after the beginning of the test, any large volume of liquid retained in the layer of the sample and particles of the drug floating at the surface of the liquid was released by rotating the cylinder about a vertical axis. The volume, Vs, occupied by the sample including any adhering mucilage was noted. The swelling capacity was obtained from the ratio of Vs to Vo. The test was performed in triplicate and the swelling capacity calculated from the mean of the three tests.

The residual moisture content was determined gravimetrically by drying a $5 \mathrm{~g}$ quantity of the Okra powders at $105{ }^{\circ} \mathrm{C}$ for 5 hours. Determinations were made in triplicate. Elemental constituents of the Okra powder were determined using $\mathrm{X}$ - ray fluorimetric analysis on the Link Analytical XR300 (Wallis Worthing, Europe). The Hausner's ratio was determined as the ratio of the initial bulk volume to the tapped volume while the Carr's index was calculated using bulk and tapped density data in equation 2 .

$$
\text { Carr's Index }=\frac{\text { Tapped density }- \text { bulk density }}{\text { Tapped density }} 100
$$

The powder porosity was computed from the bulk density and true (particle) density using the equation below:

$$
\mathrm{e}=1-\text { (bulk density / particle density) } \times 100
$$

\section{Preparation of Okra powder compacts}

Five hundred (500) $\mathrm{mg}$ of the Okra powder were compressed for 30 seconds into tablet with pre-determined loads of $28 \mathrm{~N}$, $56.5 \mathrm{~N}, 84 \mathrm{~N}, 113 \mathrm{~N}, 141 \mathrm{~N}$ and $169 \mathrm{~N}$ using a hydraulic hand press (Model C, Carver Inc., Menomomee Falls, WJ). Before each compression, the die $(12.5 \mathrm{~mm}$ in diameter $)$ and the flat faced punches were lubricated with a $2 \% \mathrm{w} / \mathrm{w}$ dispersion of magnesium stearate in $96 \%$ ethanol. After ejection, the tablets were stored over silica gel for $24 \mathrm{hrs}$ to allow for elastic recovery and hardening and to prevent false low yield values. The tablets weight and dimensions were determined within $\pm 1 \mathrm{mg}$ and $0.01 \mathrm{~mm}$ respectively. Their relative densities (D) were calculated using equation 4 .

$\mathrm{D}=\mathrm{W} /\left(\mathrm{vt} \times \mathrm{P}_{\mathrm{s}}\right)$

where $\mathrm{W}$ is the weight of the tablet $(\mathrm{g})$, vt is the volume of the tablets $\left(\mathrm{cm}^{3}\right)$ and $P_{s}$ is the solid material particle density $\left(\mathrm{g} / \mathrm{cm}^{3}\right)$. Heckel plots of $\ln (1 / 1-D)$ versus the compression pressure, $\mathrm{P}$ and Kawakita plots of $\mathrm{P} / \mathrm{C}$ vs. $\mathrm{P}$ were constructed.

\section{Compaction data analysis}

The Heckel equation is widely used for relating the relative density, $\mathrm{D}$, of a powder bed during compression to the applied pressure, $\mathrm{P}$. It is written as:

$\operatorname{In}\left(\frac{1}{1-D}\right)=K P+A$

The slope of the straight line portion, $\mathrm{K}$, is the reciprocal of the mean yield pressure, $\mathrm{P}_{\mathrm{y}}$, of the material. From the value of the intercept, A, the relative density, $\mathrm{Da}$, can be calculated using the following equation:

$D a 1-e^{-A}$

The relative density of the powder bed at the point when the applied pressure equals zero, $\mathrm{D}_{0}$, is used to describe the initial re arrangement phase of densification as a result of die filling. The relative density, $\mathrm{Db}$, describes the phase of rearrangement at low pressures and is the difference between $\mathrm{Da}$ and $\mathrm{D}_{0}$.

The Kawakita and Ludde equation is used to study powder compression using the degree of volume reduction and is written as:

$\frac{P}{C}=\frac{P}{a}+\frac{1}{a b}$

$\mathrm{C}$ is the degree of volume reduction, the constant $\mathrm{a}$ is equal to the minimum porosity of the material before compression; the constant $b$, which is termed the coefficient of compression, is related to the plasticity of the material. The reciprocal of $b$ is related to the pressure term $\mathrm{P}_{\mathrm{k}}$, which is the pressure required to reduce the powder bed by $50 \%$ (Lin and Cham, 1995). 


\section{RESULTS}

Okra powders contain $4.5 \%$ potassium, $0.11 \%$ sodium and $0.19 \%$ zinc while heavy metals like lead and mercury were absent. Chromium, cobalt, bromium and nickel were however present but in trace quantities. The Carr's Index and Hausner's ratio have become the simple and fast methods of predicting power flow characteristics (USP, 2006). As the values of these indices increase, the flow of the powder decreases. Table 1 shows that Okra powders except FOP-SD had higher values of the two indices than corn starch but lower than microcrystalline cellulose. This suggests that Okra powders except FOP-SD are more cohesive than corn starch but less cohesive than microcrystalline cellulose. The rank order of porosity is TOP-OV > MCC > OST-OV > FOP-OV > FOP-SD > CS. The particle densities of the Okra powders were higher than their corresponding bulk densities. Only FOP-SD (1.39) has similar particle density to cornstarch (1.37). The results in Table 1 showed that Okra powders had greater hydration and swelling capacities than corn starch. This implies that the rate of water uptake and the extent of water retention by the Okra powders are higher than those of corn starch. All the Okra powders had higher moisture sorption capacities compared to corn starch. FOP-SD had the highest moisture sorption capacities $(52 \%)$ while OST-OV had the lowest $(45 \%)$ value.

The Heckel plots of $\ln (1 / 1-\mathrm{D})$ versus the applied pressure for the Okra powders are shown in figure 1 . The curves for FOP-SD and corn starch shows two distinct region; a short region of rapid increase in density with increase in compression pressure (32.1 to 83.5) followed by a region of apparent linearity. This is typical of Type A materials which consolidate mainly by plastic deformation. The Heckel plots for FOP-OV, TOP-OV and OST-OV, at all compression pressures flatten out into a plateau. This resembles a Type C behavior which is characterised by initial steep-linear regions which become superimposed and flattens out as the applied pressure is increased. The three Okra brands are however without the steep-linear region. The plateau indicates that further increase in compaction load gave no further volume reduction within the compacts. This had been suggested (Adolfsson and Nystrom, 1996) to be due to infinitely small residual space available for particles to move when stressed. At this phase of extremely low porosity, the primary particles would be rigidly positioned relative to each other thereby preventing further re-positioning under pressure. The Heckel plot of FOP-OV showed some points of apparent decrease in density at pressures above $140 \mathrm{MNm}^{-2}$. This may be due to a pronounced elastic recovery or porosity expansion which resulted in expansion (Maarschalk et al., 1996). It is possible that some work of compression above pressures were stored as elastic energy (Adolfsson and Nystrom, 1996), which resulted in elastic recovery when the pressure was relieved. Such recovery could result in breakage of some bonds formed between the particles in a compact. From Table 2 FOP-SD gave the lowest value of Py (333.22) after corn starch (217.37). This implies that FOP-SD exhibits the greatest rate of plastic deformation under pressure which suggests that it may be useful

Table 1: Powder properties of Okra powders, corn starch and microcrystalline cellulose.

\begin{tabular}{|c|c|c|c|c|c|c|c|c|c|c|}
\hline Materials & $\begin{array}{c}\text { Moisture } \\
\text { Content } \\
(\%)\end{array}$ & $\begin{array}{c}\text { Moisture } \\
\text { Sorption } \\
\text { Capacity } \\
(\%)\end{array}$ & $\begin{array}{l}\text { Swelling } \\
\text { Capacity }\end{array}$ & $\begin{array}{c}\text { Hydration } \\
\text { Capacity }\end{array}$ & $\begin{array}{c}\text { Hausner's } \\
\text { Ratio }\end{array}$ & $\begin{array}{l}\text { Carr's } \\
\text { Index }\end{array}$ & $\begin{array}{c}\text { Particle } \\
\text { Density } \\
\left(\mathrm{gcm}^{-3}\right)\end{array}$ & $\begin{array}{c}\text { Bulk } \\
\text { Density } \\
\left(\mathrm{gcm}^{-3}\right)\end{array}$ & $\begin{array}{c}\text { Tapped } \\
\text { Density } \\
\left(\mathrm{gcm}^{-3}\right)\end{array}$ & $\begin{array}{c}\text { Porosity } \\
(\%)\end{array}$ \\
\hline $\begin{array}{l}\text { FOP- } \\
\text { OV }\end{array}$ & 10.9 & 47.0 & 6.40 & 7.02 & 1.32 & 24.4 & 2.02 & 0.61 & 0.81 & 69.7 \\
\hline $\begin{array}{l}\text { OST- } \\
\text { OV }\end{array}$ & 11.0 & 45.0 & 3.75 & 4.91 & 1.35 & 26.0 & 2.12 & 0.48 & 0.65 & 77.3 \\
\hline $\begin{array}{l}\text { TOP- } \\
\text { OV }\end{array}$ & 12.1 & 47.5 & 4.45 & 7.06 & 1.35 & 25.8 & 1.95 & 0.41 & 0.55 & 79.2 \\
\hline FOP-SD & 12.8 & 52.0 & 7.92 & 11.08 & 1.21 & 16.9 & 1.39 & 0.47 & 0.56 & 66.6 \\
\hline CS & 6.22 & 18.0 & 0.72 & 1.83 & 1.31 & 23.8 & 1.37 & 0.54 & 0.71 & 23.8 \\
\hline MCC & 7.20 & 18.2 & 1.63 & 2.20 & 1.53 & 34.9 & 1.42 & 0.28 & 0.43 & 78.9 \\
\hline
\end{tabular}

FopOST-OV: Okra pod stalk dried in the oven; MCC: Microcrystalline cellulose; CS: Corn starch. 


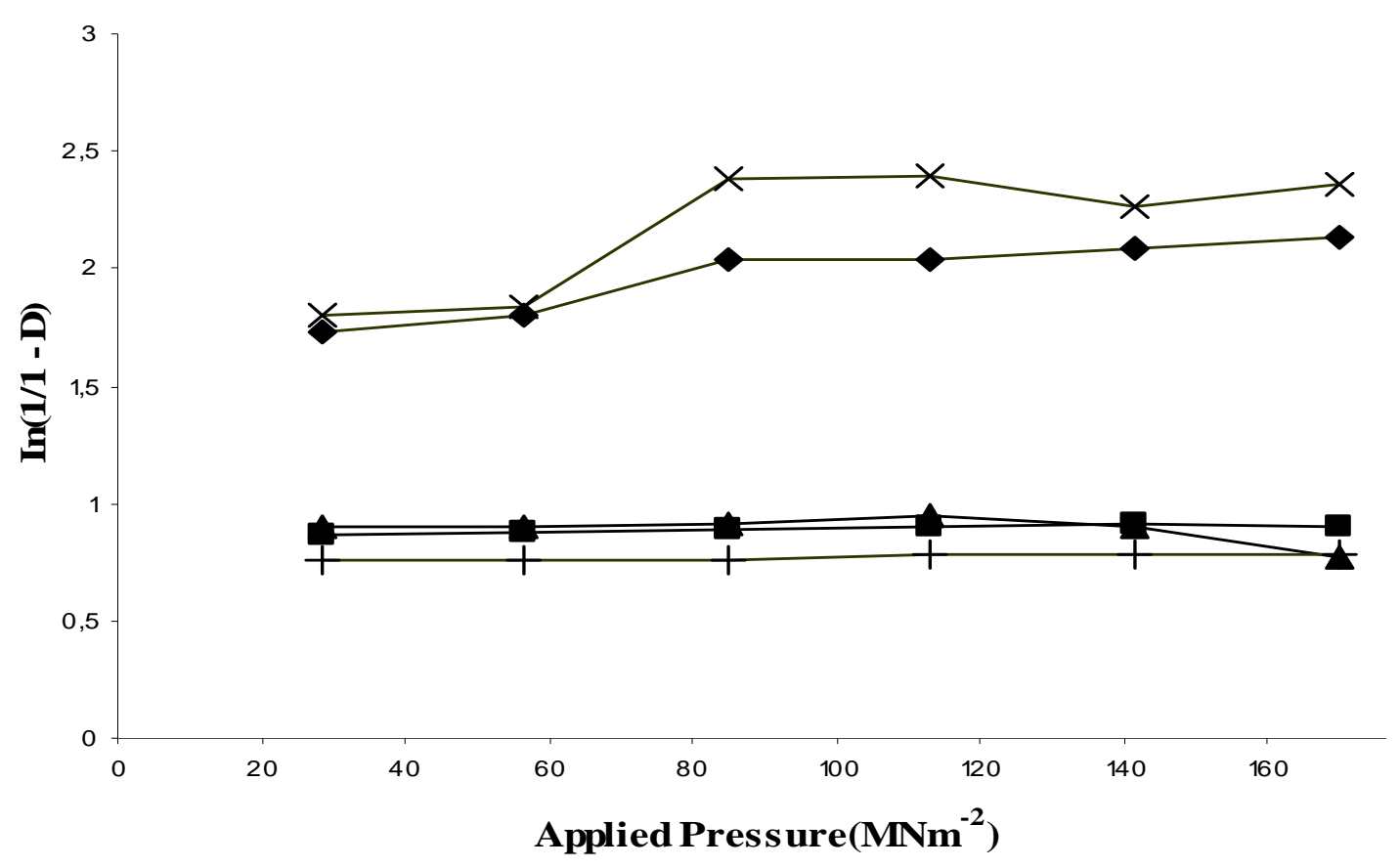

Figure 1: Heckel plots for okra powders and corn starch. $\times$, corn starch; $\bullet$ FOP-SD; $\boldsymbol{\Lambda}$, FOP-OV; $\mathbf{n}$, TOPOV; +, OST-OV.

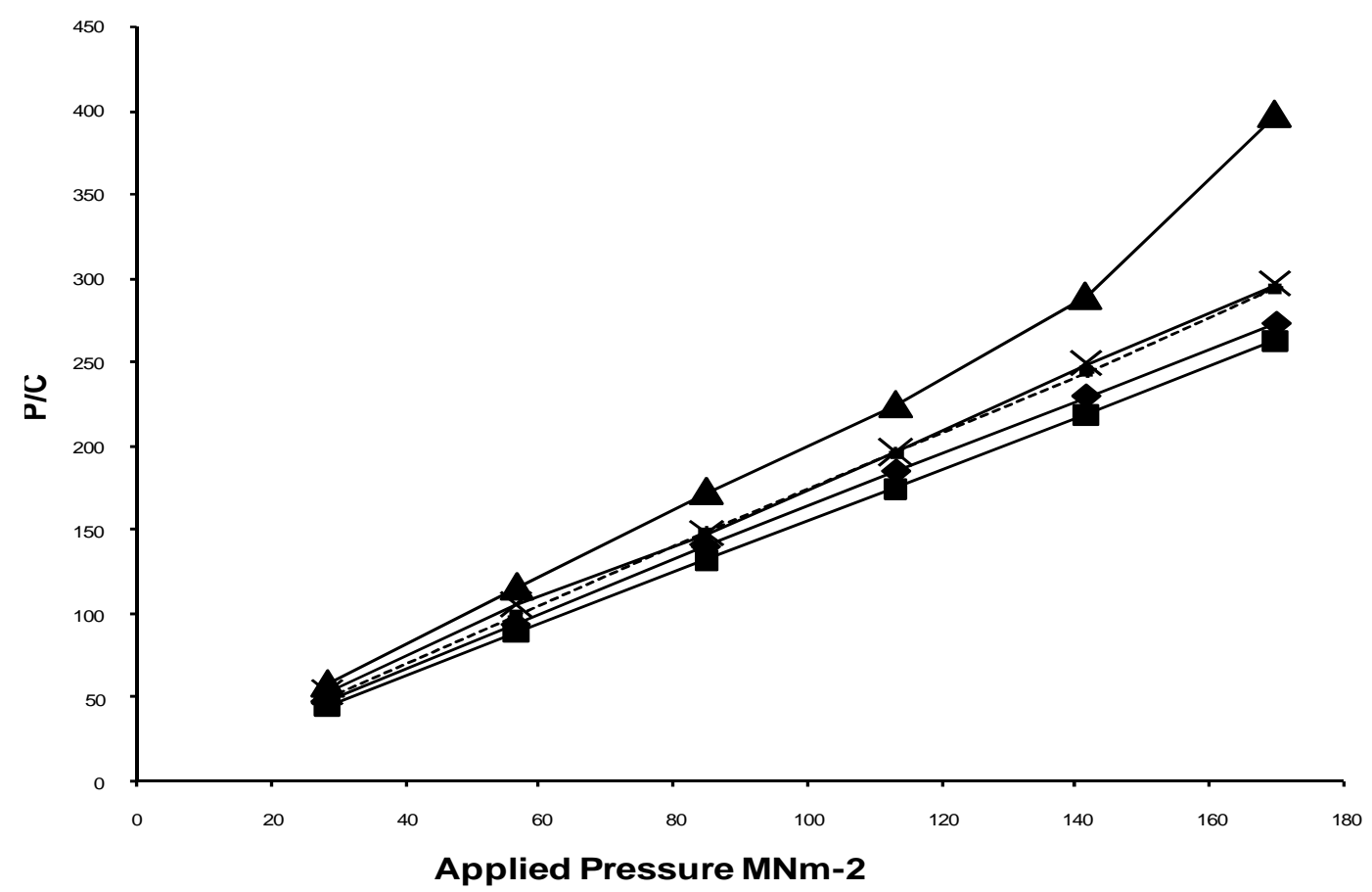

Figure 2: Kawakita plots for okra powders and corn starch. $\times$, corn starch; $\bullet$ FOP-SD; $\mathbf{\Lambda}$, FOP-OV; $\mathbf{m}$, TOPOV; +, OST-OV. 
as a direct compressible diluent. OST-OV gave the least rate. The rank order of Do is $\mathrm{CS}>$ FOP-SD $>$ FOP-OV > OST-OV > TOP$\mathrm{OV}$. The phase of rearrangement of particles in the early stages of compression i.e. under low applied pressure is represented by $\mathrm{D}_{\mathrm{b}}$. The rank order of $\mathrm{D}_{\mathrm{b}}$ values is FOP-SD > cornstarch > TOP - OV > OST - OV > FOP OV. The values of ' $a$ ' (which represent the proportion of consolidation as closest packing is attained) were obtained from the slope of the plots while values of ' $b$ ' (which represent the packing velocity) were obtained from the intercept. Smaller values of constant 'a' indicate good packing even without tapping while large values of ' $b$ ' indicate rapid packing velocity (Kawashima et al., 1984). From the table, FOP - OV $(\mathrm{a}=0.521)$ achieves good packing and has a highest packing velocity $(b=1.01)$ compared to cornstarch $(a=0.588, b=0.283)$. All the other Okra powders equally had good packing ability. The compactibility of the Okra powders which is inversely related to their $P_{k}$ values (Kawakita and Ludde, 1970/71) was of the rank order TOP - OV > FOP - OV > OST - OV > FOP - SD > cornstarch. The values of $D_{I}$ are seen to be higher than the corresponding values of Do (Table 2). The differences in the values of $D_{I}$ and $D o$ is probably due largely to the fact that while Do describes the loose initial relative density due to die filling, $D_{\text {I }}$ provides a measure of the packed initial relative density with the application of small pressure or what may be referred to as tapping of the powder (Podczeck and Sharma, 1996).

\section{DISCUSSION}

Although the Pharmacopoeial requirements are not categorical on the exact tolerable level of any possible contaminant, it should not be presumed that unusual impurities are tolerated. The British Pharmacopoeia (2005) gives limit tests for a number of possible contaminants in pharmaceutical raw materials, which may be introduced into the finished product during processing. Such tests include those for lead, arsenic, calcium, iron, potassium, aluminium, halogens and a host of others. X-ray fluorimetric (XRF) analysis of the Okra powder showed the presence of chromium, cobalt, bromium and nickel in trace amount; and sodium, potassium and zinc in levels comparable with those of the official corn starch B.P. Heavy metals like lead and mercury were however absent. The presence of heavy metals in formulated products is highly undesirable as they form stable covalent or co-ordinate complexes with body protein and can also act as catalyst (due to their variable valency state) to induce autooxidative reactions. The Pharmacopoeia has therefore placed stringent limits on the amount of lead and other heavy metals that may be present in pharmaceutical products. Table 1 shows that only FOP-SD has particle density values comparable to corn starch. The particle density influences the rate and extent of packing experienced by a material during the various unit operations of tableting such as granulation, rate of mixing, filling of dies and compression; and it is characteristic for a particular material (Itiola, 1991; Paronen and Juslin, 1983). The bulk density and the tapped density values for MCC are lower than those of CS and Okra powders. The significance of porosity on disintegration behaviour of tablets has been widely recognized (Itiola and Pilpel, 1986). Whatever the mechanism of tablet (or compact) disintegration, the initial porosity has been found to influence the rate at which the disintegration fluid penetrates the matrix of the compact or tablet and this in turn has been found to have significant impact on the

Table 2: parameters obtained from heckel and kawakita plots.

\begin{tabular}{lcccccccc}
\hline & \multicolumn{4}{c}{ Heckel Plots } & \multicolumn{5}{c}{ Kawakita Plots } \\
\cline { 2 - 9 } Materials & $\mathbf{D}_{\mathbf{0}}$ & $\begin{array}{c}\mathbf{P}_{\mathbf{y}} \\
\left(\mathbf{M N} / \mathbf{m}^{\mathbf{2}}\right)\end{array}$ & $\mathbf{D}_{\mathbf{a}}$ & $\mathbf{D}_{\mathbf{b}}$ & $\begin{array}{c}\mathbf{P}_{\mathbf{k}} \\
\left(\mathbf{M N} / \mathbf{m}^{\mathbf{2}}\right)\end{array}$ & $\mathbf{D}_{\mathbf{I}}$ & $\mathbf{a}$ & $\mathbf{b}$ \\
\hline FOP-SD & 0.334 & 333.2 & 0.802 & 0.469 & 2.216 & 0.367 & 0.633 & 0.451 \\
FOP-OV & 0.303 & 1280.1 & 0.593 & 0.290 & 0.990 & 0.479 & 0.521 & 1.010 \\
TOP-OV & 0.208 & 2814.5 & 0.573 & 0.365 & 0.909 & 0.351 & 0.649 & 1.100 \\
OST-OV & 0.227 & 3203.1 & 0.523 & 0.296 & 1.168 & 0.416 & 0.584 & 0.856 \\
CS & 0.395 & 217.4 & 0.814 & 0.419 & 3.528 & 0.412 & 0.588 & 0.283 \\
\hline
\end{tabular}


subsequent disintegration rate (Lowenthal and Wood, 1973). The Okra pod powders were more porous than corn starch but less porous than microcrystalline cellulose except TOP$\mathrm{OV}$, which had porous than miccocrystalline cellulose. This suggest that the rate water uptake and swelling may be more in Okra powders than corn starch but less than that of microcrystalline cellulose. Hence, Okra powders may be a better disintegrant than corn starch but may not be as good as microcrystalline cellulose. The flow properties of a powder are essential in determining its suitability as a direct compression excipient. The angle of repose, Hausner's ratio and Carr's index are considered as indirect measurement of powder flowability (Staniforth, 1996). As the values of these indices increase, the flow of the powder decreases. In general, however, Hausner's ratio greater than 1.25 indicates poor flow; Carr's index below $16 \%$ indicates good flowability while values above $35 \%$ indicate cohesiveness. In this study, the values of Hausner's ratio and Carr's Index showed that the Okra powders had poor flow except FOPSD which had fairly good flow. Consequently a glidant will be needed when they are to be used in solid dosage production processes. The Hausners ratio previews the degree of densification which could occur during tableting. The higher the ratio, the greater the propensity of the powder to densify (Ohwoavworhua and Adelakun, 2005). The result showed that Okra powders densified more than corn starch but less than microcrystalline cellulose.

Swelling which is generally accepted as an indication of tablet disintegration ability can be assessed by the determination of hydration capacity, swelling capacity and moisture sorption profile (Ohwoavworhua and Adelakun, 2005). The hydration capacity values obtained for the Okra powders were greater than those of microcrystalline cellulose and corn starch (Table 1). OST - OV with the lowest hydration capacity of 4.91 is capable of absorbing about five times its own weight of water, approximately thrice more water than corn starch and about twice more water than microcrystalline cellulose. The swelling capacity values of Okra powders, which reflects the increase in volume of Okra powders following water absorption is greater than the values for corn starch and microcrystalline cellulose. Thus, if Okra powder was incorporated in tablet formulation as a disintegrant it would probably produce tablet disintegration by two mechanisms: capillary or wicking due to interparticulate water and swelling. The superior hydration and swelling capacities of the Okra powders $(p<0.05)$ would suggest that they may likely be better disintegrant than corn starch and microcrystalline cellulose. However, it has been observed that hydration and swelling capacity may not be absolute indices of disintegrant efficacy (Adebayo and Itiola, 1998b). Nevertheless, they are still considered as useful qualitative assessment of potential disintegrating agents. The absorption of moisture by solid dosage forms and excipients provide information for selecting excipients and for determining the humidity control required during production and storage. The amount of moisture absorbed by drugs and excipients affects the flow, compression characteristics and hardness of tablets. The moisture sorption capacity of the Okra pods was significantly $(p<0.01)$ higher than the values obtained for corn starch and microcrystalline cellulose. In addition, the moisture contents of the Okra powders were high. The formation of film of moisture on the Okra powder surface may reduce friction at the die wall by acting as an internal lubricant. It may also increase the ease with which the individual particles can slip and flow during compression. However, it is important that the moisture content be kept as low as possible during storage to prevent microbial spoilage, hydrolysis and enzymatic decomposition. Consequently, Okra powders would need to be dried further if it is to be used as an excipient in the formulation of hydrolysable drugs such as aspirin. In addition, Okra powders should be stored in air-tight containers due to their susceptibility to moisture sorption at atmospheric condition.

The compressional characteristics of the Okra pod powders were evaluated using Heckel and Kawakita plots, and density measurements. Each of the two plots has its limitations. Heckel plot is known to generally exhibit linearity at high pressures while Kawakita plots generally exhibit linearity at low pressures (Celik, 1992). The use of two equations together in this study gave a more 
comprehensive understanding of the compression characteristics of the Okra powders. The shape of Heckel plot for FOPSD is typical of Type A materials, which consolidate mainly by plastic deformation. FOP-OV, TOP-OV and OST-OV resemble Type $\mathrm{C}$ materials which consolidate mainly by plastic deformation and asperitic melting. The mean yield pressure, $\mathrm{P}_{\mathrm{Y}}$ is inversely related to the ability of the material to deform plastically under pressure and it gives an impression of the ease of plastic deformation and softness of the material i.e. soft, ductile powders have lower yield values. A low value tends to favour plastic deformation during compaction as a result of the rebonding of smaller primary crystals (Deodhar et al., 1998) and also reflects low resistance to pressure, good densification and easy compression (Jivraj et al., 2000). The rank order of Py value was corn starch < FOP-SD <FOP-OV < TOP-OV $<$ OST-OV. This study shows that corn starch had higher plasticity than the Okra powders. Of all the Okra powders, only FOP-SD had $\mathrm{P}_{\mathrm{y}}$ value comparable to that of corn starch and may therefore be useful as a direct compression excipient. Other Okra powders have very high $\mathrm{P}_{\mathrm{y}}$ values indicating hardness and high resistance to compression. The $\mathrm{P}_{\mathrm{k}}$ values (obtained from the Kawakita plots), which are an inverse measurement of the plastic deformation occurring during the compression process were generally lower for the Okra powders than for corn starch. Thus, corn starch exhibited a lower degree of total plastic deformation during the compression process. Among the Okra powders, TOP-OV had the highest compactibility (i.e. total plastic deformation) followed by FOP-OV, then OST$\mathrm{OV}$ and finally FOP-SD. It has been established that the lower the $\mathrm{P}_{\mathrm{k}}$ value, the more the total plastic deformation that occurs during compression. Although corn starch exhibited a faster onset of plastic deformation than Okra powders during compression, as indicated by the low $\mathrm{P}_{\mathrm{y}}$ values, it also exhibited the lowest degree of plastic deformation during compression. On the other hand, Okra powders exhibited a slower onset of plastic deformation but a higher amount of plastic deformation during compression. With the phenomenon of plastic deformation being time dependent (Akande et al., 1998) it should be of significant benefit to use a long dwell time for powders with a combination of high $\mathrm{P}_{\mathrm{y}}$ but low $\mathrm{P}_{\mathrm{k}}$ while materials with a high $\mathrm{P}_{\mathrm{y}}$ and $P_{k}$ values would give compressional problems on virtually any type of tableting machine and the addition of plastic material may be necessary in such cases. Thus, corn starch will be more appropriate than the Okra powders on a high speed tablet machine with a short dwell time while a long dwell time will be preferable for Okra powders. The density of the powders at zero pressure $\left(D_{0}\right)$ was highest for corn starch followed by FOP-SD, FOP-OV and TOP-OV respectively. The $\mathrm{D}_{\mathrm{o}}$ value represents the degree of initial packing in the die as a result of die filling. This result indicates that cornstarch exhibited a higher degree of packing in the die because of die filling than the Okra powders. The $D_{b}$ value represents the particle rearrangement phase in the early compression stages and tends to indicate the extent of particle fragmentation although fragmentation can occur concurrently with plastic and elastic deformation of constituent particles. All the Okra powders (except FOP-SD) had lower $D_{b}$ value compared to corn starch. The high $D_{b}$ value of FOP-SD may be due to particle desegmentation/shearing off of small individual particles. The values of $D_{b}$ were higher than those of $D_{0}$ because of particle fragmentation and the subsequent filling of void spaces between the particles which occurs extensively at low pressures to facilitate densification. The loose packing of the large particles at zero pressure tended to yield low $\mathrm{D}_{\mathrm{o}}$ values. Similar observations were made by Itiola and Pilpel, (1986a) while studying the tableting characteristics of metronidazole tablet formulations. The $\mathrm{D}_{\mathrm{I}}$ values were higher than the $D_{0}$ values. Thus, the results suggest that while $D_{0}$ values described the loose initial relative density of the formulation due to die filling, the values of $D_{\text {I }}$ provides a measure of the packed initial relative density with the application of small pressure.

This study reveals that okra powder possesses good physicochemical and compaction properties which could be explored in its development as an excipient in tablet formulation and production.

\section{REFERENCES:}

Adebayo AS, Itiola OA. 1998a. Evaluation of breadfruit and cocoyam starches as 
exodisintegrant in a paracetamol tablet formulation. Pharm. Pharmacol. Commun., 4: 385-389.

Adolfsson A, Nystrom C. 1996. Tablet strength, porosity, elasticity and solid state structure of tablets compressed at high loads. Int. J. Pharm., 132: 95-106.

Akande OF, Ford JL, Rowe PH, Rubinstein MH. 1998. The effects of lag time and dwell time on the compaction properties of 1:1 paracetamol/microcrystalline cellulose tablets prepared by precompression and main compression. $J$. Pharm. Pharmacol., 50: 19-28.

British Pharmacopoeia. 2005. (Vol. 2). Crown Copyright: London; 2185-2186.

British Standard 1460. 1970. British Standard Institution: London.

Celik M. 1992. Overview of compaction data analysis techniques. Drug Dev. Ind. Pharm., 18: 767-810.

Deodhar UP, Paradkar AR, Purohit, AP. 1998. Preliminary evaluation of Leucaena leucocephala seed gum as a tablet binder. Drug Dev. Ind. Pharm., 24: 577-582.

Gohel MC, Jogani PD. 2005. A review of coprocessed directly compressible excipients. J. Pharm. Pharma. Sci., 8(1): 7693.

Itiola OA, Pilpel N. 1986. Studies on metronidazole tablet formulation. $J$. Pharm. Pharmacol., 38: 81-86.

Itiola OA, Pilpel N. 1986a. Tableting characteristics of metronidazole formulations. Int. J. Pharm., 31: 99 - 105.

Itiola OA. 1991. Compressional characteristic of three starches and the mechanical properties of their tablets. Pharm. World J., 8: 91-94.

Jivraj M, Martini LG, Thomson CM. 2000. An overview of the different excipients useful for the direct compression of tablets. PSTT, 3: 58-63.

Kawakita K, Ludde KH. 1970/71. Some considerations on powder compression equations. Powder Technol., 4: 61-68.

Kawashima Y, Okumura M, Kojima A. 1984. Direct preparation of spherically agglomerated salicylic acid crystal during crystallization. J. Pharm. Sci., 73: 15351538.

Leuenberger H, Rohera B. 1986. Fundamental properties of powder compression I. The compactibility and compressibility of pharmaceutical powders. Pharm. Res., 3: 12-22.

Lin C, Cham T. 1995. Compression behaviour and tensile strength of heat treated polyethyleneglycols, Int. J. Pharm., 118: 169-179.

Lowenthal N, Wood JH. 1973. Mechanism of action of starch as a tablet disintegrant: Location and structure of starch in tablets. J. Pharm. Sci., 62: 287.

Maarschalk KV, Zuurman K, Vromans H, Bolhuis GK, Lerk CF. 1996. Porosity expansion of tablets as a result of binding and deformation of particulate solids. Int. J. Pharm., 140: 185-193.

Ohwoavworhua F, Adelakun TA. 2005. Some physical characteristics of microcrystalline cellulose obtained from raw cotton of Cochlospermum planchonii. Tropical Journal of Pharm. Research., 4(2): 501-507.

Paronen P, Juslin M. 1983. Compressional characteristics of four starches. J. Pharm. Pharmacol., 35: 627-635.

Patel S, Kaushal A, Bansal A. 2006. Effect of particle size and compression force on compaction behaviour and derived mathematical parameters of compressibility. Pharm. Res. Pharmacol., 28(2): 75-81.

Podczeck F, Sharma M. 1996. The influence of particle size and shape of components of binary powder mixtures on the maximum volume reduction due to packing. Int. J. Pharm., 134: 41-47.

Smith P, Polomsky B, Shaughnessy D. Okra Home and Garden Information Center, Clemson University http:// hgic. Clemson.edu/factsheet/HG1C1313.htm. Accessed 17 December, 2006.

Staniforth JN. 1996. Powder flow. In Pharmaceutics: The Science of Dosage form Design, Aulton ML (ed). ELBS; 600-615. 